\title{
Cómputo simbólico y gráfico: estudio del sistema masa-resorte
}

\author{
Graphic and symbolic computation: Analysis on the spring-mass system \\ M. A. López-Mariño*1, J. A. Hernández-Olvera ${ }^{2}$, L. A. Barroso², J. C. Trujillo Caballero ${ }^{2,3}$ \\ ${ }^{1}$ Escuela de Ingeniería y Ciencias, Tecnológico de Monterrey, Córdoba, Veracruz, México \\ ${ }^{2}$ Departamento de Ingeniería, Tecnológico de Monterrey, Córdoba, Veracruz, México \\ ${ }^{3}$ Departamento de Ingeniería Eléctrica, Instituto Tecnológico de Orizaba, Orizaba, Veracruz, México
}

Received on August 1, 2016. Revised on September 27, 2016. Accepted on September 28, 2016.

\begin{abstract}
En este trabajo modelamos y simulamos el movimiento oscilatorio de un sistema masa-resorte mediante la solución, simbólica y gráfica, de sus ecuaciones diferenciales con Maple y la comparamos con la solución analítica. Nuestro propósito es ofrecer a los estudiantes una guía práctica para el análisis de problemas de naturaleza ondulatoria utilizando las capacidades de este programa como herramienta computacional. Presentamos, como apéndices, las instrucciones necesarias para obtener los resultados presentados. Discutimos las ventajas didácticas que ofrece el paquete en la solución de este tipo de problemas.

Palabras clave: Ecuaciones Diferenciales, Sistema masa-resorte, Modelación y Simulación Computacional.
\end{abstract}

In this work we model and simulate the oscillating movement of a system of spring-mass by solving, symbolically and graphically, their differential equations with Maple and compare them to the analytic solution. Our purpose is to offer students a practical guide for the analysis of problems of wave nature using this program computational tool's capabilities. The necessary commands to obtain the shown results are provided. At the same time, the computational tool's didactic advantages are analyzed for this type of movement.

Keywords: Differential Equations, Spring-mass system, Computational Modeling and Simulation.

\section{Introducción}

Los cursos de ecuaciones diferenciales (ED) que se ofrecen en las carreras de ingeniería, pretenden sentar las bases matemáticas para el tratamiento de aplicaciones en áreas diversas de la ingeniería, mediante la solución de ecuaciones diferenciales ordinarias (EDO). Las aplicaciones son variadas [1-8], pero, en algunos casos, los alumnos tienden a formular o basarse en procedimientos establecidos para encontrar la solución a problemas de sistemas físicos $[9,10,11,12]$. El tiempo que invierten los estudiantes en la generación de resultados de manera analítica es muy valioso; sin embargo, pensamos que el proceso de aprendizaje de las EDO se facilitaría, en gran medida, si los conceptos nuevos se

*Endereço de correspondência: malm@itesm.mx enfocan desde diferentes puntos de vista. Por esta razón, buscamos promover, en los estudiantes, el uso de herramientas computacionales con las cuales puedan obtener soluciones numéricas, simbólicas y gráficas de las EDO. La alternativa computacional que elegimos fue el paquete Maple, que se alinea con las expectativas de aprendizaje que tenemos para nuestros estudiantes.

En Maple, la solución de una EDO se puede generar como en el ejemplo que mostramos a continuación:

Si la ecuación que deseamos resolver es

$$
y^{\prime}+y \sin (x)=0,
$$

usamos la instrucción (al ejecutar cada instrucción, Maple devuelve lo establecido o solicitado)

$$
>g:=y(x) * \sin (x) ;
$$




$$
g:=y(x) \sin (x)
$$

y luego formamos la ecuación deseada a la que llamamos "ode"

$$
\begin{gathered}
>\text { ode }:=\operatorname{diff}(y(x), x)+g \\
\text { ode }:=\left(\frac{d}{d x} y(x)\right)+y(x) \sin (x)
\end{gathered}
$$

La ecuación se soluciona como sigue y asignamos el resultado a una variable "soln"

$$
\begin{gathered}
>\operatorname{soln}:=\text { dsolve }(\text { ode, } y(x)) ; \\
\text { soln }:=y(x)=\_C I e^{\cos (x)}
\end{gathered}
$$

En la Fig. 1, presentamos la solución gráfica de la ecuación (1) para diferentes valores de la constante $C_{1}$.

Como vemos, es posible plantear una EDO de manera sencilla y obtener su solución general, así como también la gráfica de algunas soluciones particulares.

En nuestros cursos de ED, la estrategia que establecimos incluye el Aprendizaje Orientado a Proyectos (del inglés Project Oriented Learning, POL) para que, de manera colaborativa, los estudiantes incursionen en la modelación y la simulación de sistemas físicos que impliquen el planteamiento y solución de EDO.

Una vez integrados los equipos de trabajo, les asignamos un proyecto relacionado con un sistema

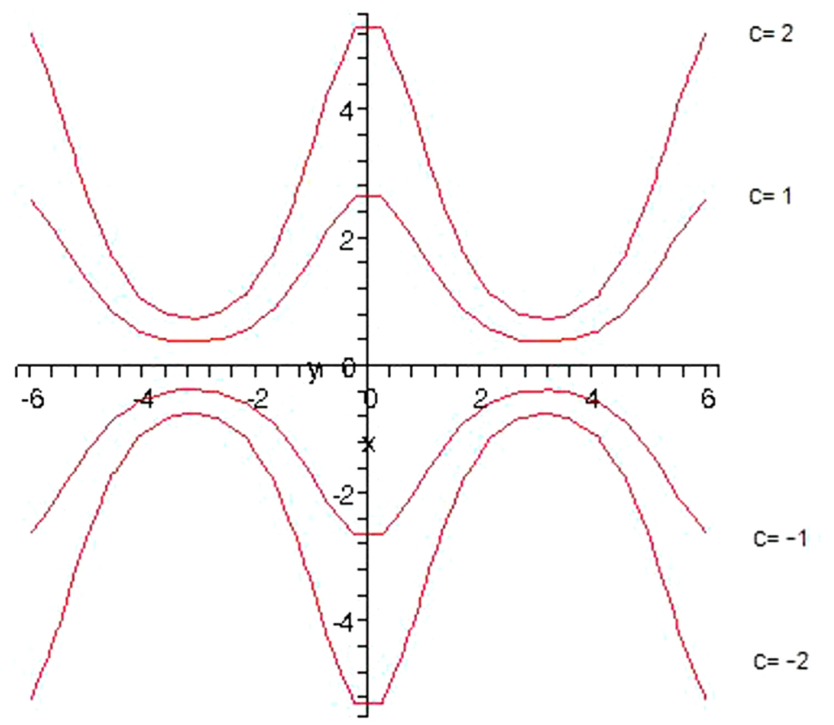

Figura 1: Gráfica de algunos miembros de la solución. Los valores de $c$ se colocaron por edición de la gráfica y no por Maple. que ya conocían de sus cursos de física; ahora debían modelarlo con EDO y simular su comportamiento bajo condiciones específicas. Para completar ese proceso, y dado que nuestros estudiantes no sabían usar Maple, les proporcionamos algunas instrucciones necesarias para realizar su trabajo; las necesarias para obtener los resultados que presentamos se encuentran en los Apéndices A y B.

En este trabajo, mostramos el caso desarrollado en un curso de ED, donde se aprovecharon los recursos del paquete Maple [13,14]: el análisis de un sistema autónomo modelado por una EDO del tipo

$$
y^{\prime}=h(y)
$$

en cuyo miembro derecho no se encuentra, explícitamente, la variable independiente. Particularmente, estudiamos el sistema masa-resorte, mediante la solución de las EDO que resultan para el movimiento armónico simple y para el amortiguado. Analizamos los aspectos discutidos, de posición y velocidad, en los libros de texto de física de las carreras de ingeniería $[9,10,11] \mathrm{y}$, además, mediante sus diagramas de fase [12]. Con el propósito de hacer más fácil el análisis para nuestros estudiantes (y posibles lectores), presentamos, con detalle, los argumentos conocidos $[9,10,11,12]$.

En la sección 2, analizamos el movimiento del oscilador armónico simple. En la sección 3, estudiamos el movimiento del oscilador amortiguado. Luego, en la sección 4, comentamos acerca de las ventajas de trabajar este tipo de problemas con herramientas computacionales como Maple.

\section{Oscilador armónico simple}

El problema del sistema masa-resorte es muy común y se encuentra en la mayoría de los libros de ED, [1-8]. Se supone un objeto de masa $M$ atada a un resorte (con masa despreciable) de constante elástica $k$, cuyo movimiento está restringido a una dimensión como se presenta en la Fig. 2.

En nuestro caso, el objeto se suelta, desde el reposo, cuando el resorte se encuentra en su máxima elongación y, entonces, empieza a oscilar; la única fuerza que actúa sobre la masa es la que ejerce el resorte, $F=k x$, en dirección contraria al movimiento, como se aprecia en la Fig. 3.

La EDO de segundo orden con coeficientes constantes que modela el movimiento del objeto de masa 


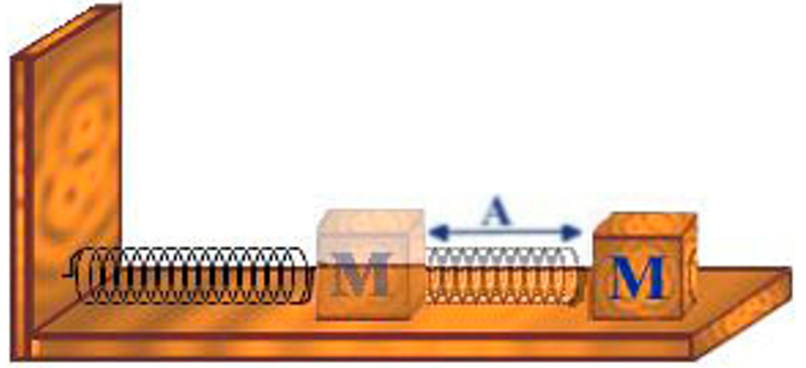

Figura 2: Sistema masa-resorte.

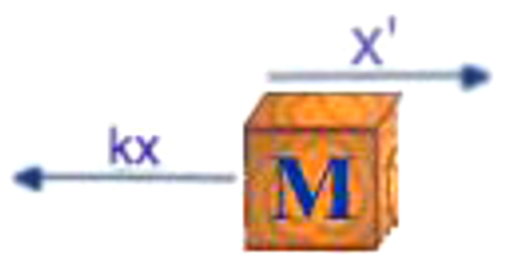

Figura 3: Diagrama de cuerpo libre de la masa.

$M$ es

$$
x^{\prime \prime}+\frac{k}{M} x=0
$$

y sus condiciones iniciales están dadas por $x(0)=A$ y $x^{\prime}(0)=0$. La solución general de la ec. (3) queda como

$$
x(t)=C_{1} \cos \omega t+C_{2} \sin \omega t ;
$$

si tomamos en cuenta las condiciones iniciales, la solución anterior se expresa como

$$
x(t)=A \cos \omega t .
$$

Con los valores para las constantes: $M=10 \mathrm{Kg}, k=$ $5 \mathrm{~N} / \mathrm{m}$ y $A=5 \mathrm{~m}$, la función de posición presentada por Maple es

$$
x(t)=5 \cos \left(\frac{1}{2} \sqrt{2} t\right)
$$

y la Fig. 4 muestra su comportamiento; inicia en su máxima posición, como supusimos, y es periódica con amplitud constante, pues en el sistema no existen fuerzas disipativas.

Si se deriva (5) con respecto al tiempo, se obtiene la función de la velocidad del objeto

$$
x^{\prime}(t)=-\omega A \sin \omega t ;
$$

con los mismos valores, Maple establece

$$
\frac{d}{d t} x(t)=-\frac{5}{2} \sin \left(\frac{1}{2} \sqrt{2} t\right) \sqrt{2} .
$$

La gráfica se presenta en la Fig. 5. La velocidad inicia en su valor cero, condición inicial, y luego toma

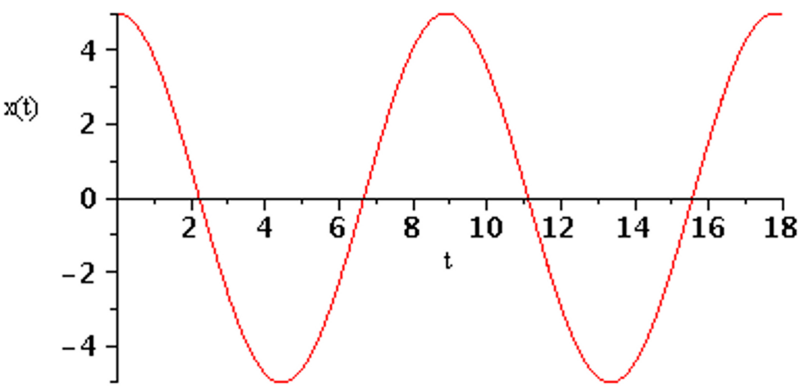

Figura 4: Posición de la masa.

valores negativos, que corresponden al movimiento de la masa desde su máxima elongación a su punto de equilibrio, en que su velocidad es máxima hacia la izquierda. Llega a su máxima compresión y, luego, regresa a su punto de partida.

Otra forma de analizar este problema es a través de los diagramas de fase del movimiento [12]. Para obtenerlos, se parte de las ecuaciones paramétricas (5) y (7) y se usa la identidad trigonométrica

$$
\operatorname{sen}^{2} \theta+\cos ^{2} \theta=1
$$

se obtiene

$$
\frac{x^{2}}{A^{2}}+\frac{x^{\prime 2}}{\omega^{2} A^{2}}=1,
$$

que es, precisamente, la ecuación de una elipse que representa el diagrama de fase del movimiento en el plano $x x^{\prime}$. Esta figura se genera para una condición particular de la energía total, que en este caso está dada por

$$
E=\frac{1}{2} k A^{2}
$$

se puede apreciar esto si se toma en cuenta la condición (11) y el valor de $\omega^{2}$ para reescribir la ec. 10. como

$$
\frac{x^{2}}{2 E / k}+\frac{x^{\prime 2}}{2 E / M}=1 .
$$

La Fig. 6 muestra el diagrama de fase del oscilador armónico simple para diferentes energías $[8,12]$ obtenido por Maple.

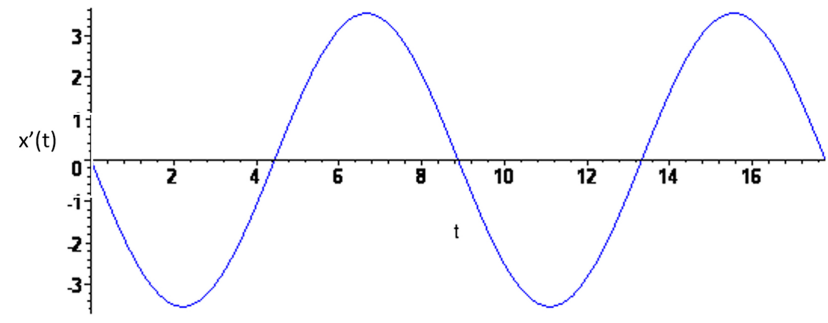

Figura 5: Velocidad de la masa. 
En realidad, la expresión de la ec. (12) representa un conjunto de elipses para diferentes energías, que corresponden a las distintas fases del diagrama. Son trayectorias cerradas, pues el movimiento es armónico con período $\frac{2 \pi}{\omega}$, en dirección de las manecillas del reloj. Si el movimiento se inicia cuando $x=5 \mathrm{~m}$ $\mathrm{y}$, en ese instante, su velocidad es $v=0$ (Fig. 2), se alcanza su máxima velocidad, $\frac{5}{2} \sqrt{2} \frac{\mathrm{m}}{\mathrm{s}}$, en dirección negativa de las $x$ cuando llega a la posición de equilibrio $x=0$; continúa hasta frenarse al llegar a $x=-5 m$ Luego, cambia de dirección el movimiento del objeto y alcanza su máxima velocidad, $\frac{5}{2} \sqrt{2} \frac{m}{s}$, al pasar por $x=0$; luego se frena al concluir el ciclo.

\section{Oscilador amortiguado}

A diferencia del sistema armónico, este tipo de movimiento cuenta con un amortiguamiento [1, 3, 12], que, usualmente, es proporcional a la velocidad de la masa; es decir, $c x^{\prime}$. Con esto, la EDO de segundo orden y coeficientes constantes que modela al sistema se escribe como

$$
x^{\prime \prime}+\frac{c}{M} x^{\prime}+\frac{k}{M} x=0,
$$

y tiene condiciones iniciales, como en el caso anterior, dadas por $x(0)=A$ y $x^{\prime}(0)=0$; la ecuación característica de 13 es

$$
\lambda^{2}+\frac{c}{M} \lambda+\frac{k}{M}=0,
$$

donde es usual considerar $\beta=\frac{c}{2 m}$ y $\omega_{0}^{2}=\frac{k}{M} ; \beta$ es el parámetro de amortiguamiento y $\omega_{0}$ es, precisamente, la frecuencia del oscilador armónico, llamada frecuencia natural del sistema

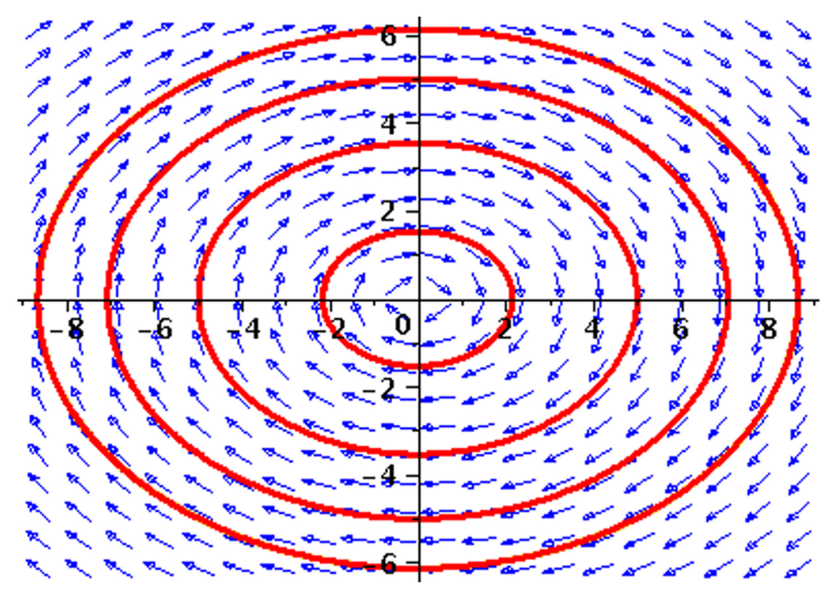

Figura 6: Diagrama de fase del Movimiento Armónico Simple.
De (14), se obtienen las raíces

$$
\lambda=-\beta \pm \sqrt{\beta^{2}-\omega_{0}^{2}},
$$

y se desprenden 3 posibilidades que a continuación se estudian:

\subsection{Oscilador submortiguado}

Cuando $\beta<\omega_{0}$, se dice que el sistema es subamortiguado y su función de posición queda expresada como

$$
x(t)=e^{-\beta t}\left(C_{1} \cos \left(\omega_{d} t\right)+C_{2} \sin \left(\omega_{d} t\right)\right)
$$

donde

$$
\omega_{d}=\sqrt{\beta^{2}-\omega_{0}^{2}},
$$

es la frecuencia del sistema con amortiguamiento.

La expresión para $x(t)$, también se puede reescribir como

$$
x(t)=A e^{-\beta t} \sin \left(\omega_{d} t+\delta_{0}\right)
$$

y la respuesta de Maple con los valores de $M=20 \mathrm{~kg}$, $k=20 \mathrm{~N} / \mathrm{m}, A=5 \mathrm{~m}$ y $c=5 \mathrm{~kg} / \mathrm{s}$ es

$$
\begin{aligned}
x(t) & =\frac{5}{21} e^{-\frac{1}{8} t}\left(\sin \left(\frac{3}{8} \sqrt{7} t\right) \sqrt{7}\right. \\
& \left.+21 \cos \left(\frac{3}{8} \sqrt{7} t\right)\right)
\end{aligned}
$$

Como 18 contiene el factor $e^{-\beta t}$ para $\beta>0$, los desplazamientos de la masa se vuelven insignificantes a medida que el tiempo transcurre, como puede verse en la Fig. 7. Para este tipo de movimiento, en que el amortiguamiento es débil, se pueden calcular las trayectorias del diagrama de fase de manera similar al oscilador armónico simple, por lo que es necesario determinar la expresión de la velocidad que se obtiene al derivar 18

$$
\begin{array}{r}
x^{\prime}(t)=-A e^{-\beta t}\left(\beta \sin \left(\omega_{d} t+\delta_{0}\right)\right. \\
\left.-\omega_{d} \cos \left(\omega_{d} t+\delta_{0}\right)\right) ;
\end{array}
$$

como la masa parte del reposo, $x^{\prime}(0)=0$, podemos determinar que

$$
\delta_{0}=\tan ^{-1}\left(\frac{\omega_{d}}{\beta}\right)
$$


Para las condiciones dadas, la función de velocidad está dada por Maple como

$$
\frac{d}{d t} x(t)=-\frac{5}{168} e^{-\frac{1}{8} t}\left(\sin \left(\frac{3}{8} \sqrt{7} t\right) \sqrt{7}+21 \cos \left(\frac{3}{8} \sqrt{7} t\right)\right)+\frac{5}{21} e^{-\frac{1}{8} t}\left(\frac{21}{8} \cos \left(\frac{3}{8} \sqrt{7} t\right)-\frac{63}{8} \sin \left(\frac{3}{8} \sqrt{7} t\right) \sqrt{7}\right)
$$

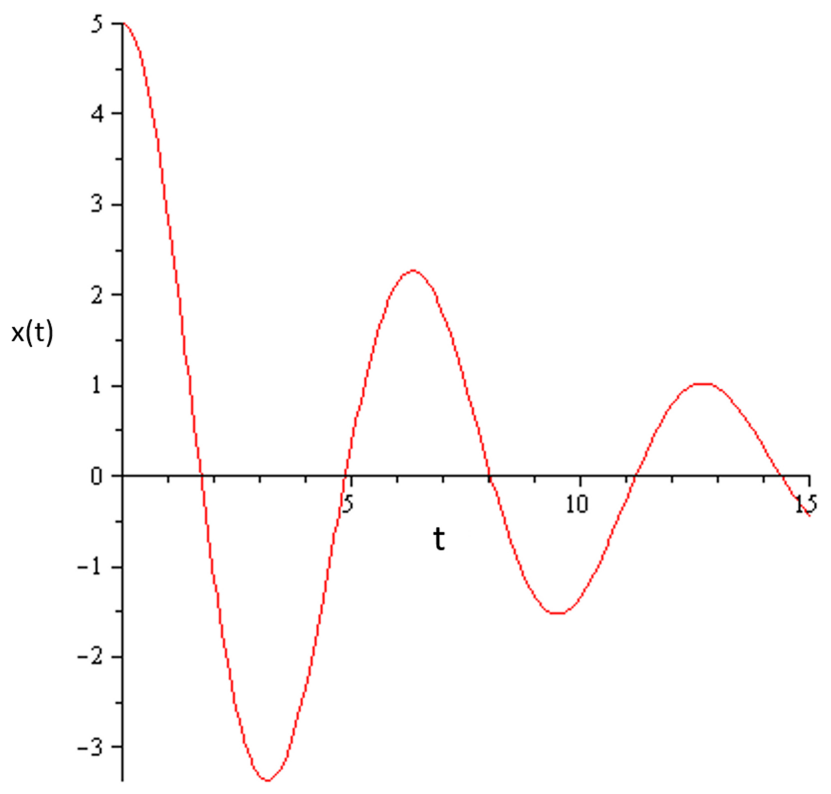

Figura 7: Posición del movimiento subamortiguado.

y su gráfica se presenta en la Fig. 8. Se observa que la velocidad de la masa es negativa después del reposo y corresponde a su movimiento hacia su posición de máxima compresión del resorte y luego toma valores en dirección positiva hacia la posición inicial.

Para obtener el diagrama de fase de este movimiento, no se puede eliminar el parámetro $t$ con la facilidad con que se hizo en el caso del oscilador armónico; se asume que

$$
\begin{gathered}
\rho=A e^{-\beta t}, \\
\theta=\omega_{d} t+\delta_{0}
\end{gathered}
$$

y luego se escriben $(18)$ y 20 como

$$
\begin{gathered}
x(t)=\rho \sin \theta \\
x^{\prime}(t)=-\rho\left(\beta \sin \theta-\omega_{d} \cos \theta\right)
\end{gathered}
$$

y se ejecuta la transformación lineal entre 250 y (26) dada por

$$
y(t)=x^{\prime}(t)+\beta x(t)
$$

que se reduce a

$$
y(t)=\omega_{d} \rho \cos \theta
$$

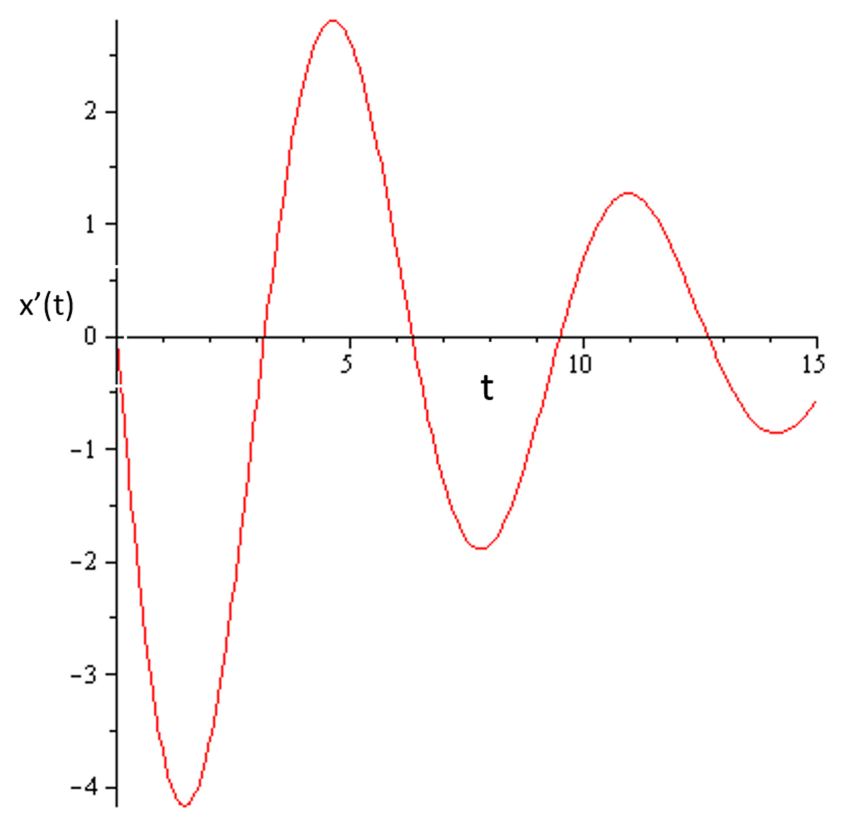

Figura 8: Velocidad del movimiento subamortiguado.

Por último, se elevan al cuadrado (25) y 28) y con ayuda de la ec. (9) se obtiene

$$
\frac{x^{2}}{\rho^{2}}+\frac{y^{2}}{\rho^{2} \omega_{d}^{2}}=1 .
$$

Aunque esta ecuación es idéntica, en forma, a la ec. (12), se debe tener en mente que la variable $y(t)$ es una combinación lineal entre $x(t)$ y $x^{\prime}(t) \mathrm{y}$, por lo tanto, 29p contiene los puntos $(x, y)$ de un espacio fase modificado. Como $x(t)$ e $y(t)$ contienen a $\rho$, que es función de una exponencial negativa, la trayectoria en este espacio es una elipse cuyos ejes decrecen exponencialmente con el tiempo y su punto de inicio es $x_{0}=A \sin \delta_{0}$. El comportamiento de una trayectoria en el plano $x x^{\prime}$ puede verse para un amortiguamiento débil con factor $\beta$, pequeño comparado con la frecuencia angular de un oscilador sin amortiguamiento, donde se considera que

$$
\begin{aligned}
& \omega_{d} \approx \omega_{0} \\
& y \approx x^{\prime}
\end{aligned}
$$

con esto, la ec. $(29)$ se transforma en

$$
\frac{x^{2}}{\rho^{2}}+\frac{x^{\prime 2}}{\rho^{2} \omega_{0}^{2}}=1
$$


La siguiente gráfica, Fig. 9, generada por Maple representa el diagrama de fase para el movimiento subamortiguado.

La energía de este oscilador decae exponencialmente con el tiempo y a esto corresponde la naturaleza de espiral que la trayectoria refleja. Esto puede verificarse de las ecs. (25) y (28), ya que ambas dependen de $\rho$, ec. 23), cuya naturaleza es la de una exponencial negativa.

\subsection{Oscilador críticamente amortiguado}

Cuando $\beta=\omega_{0}$, se dice que el sistema está críticamente amortiguado y su función de posición está dada por

$$
x(t)=(A t+B) e^{-\beta t}
$$

y con valores de $M=20 \mathrm{~kg}, A=5 \mathrm{~m}, k=20 \mathrm{~N} / \mathrm{m}$ y $c=40 \mathrm{~kg} / \mathrm{s}$, el resultado de Maple es

$$
x(t)=5 e^{-t}(1+t)
$$

su gráfica se presenta en la Fig. 10, donde se observa que el movimiento se amortigua, completamente, justo antes de llegar a la posición de equilibrio.

La función de velocidad se expresa por

$$
x^{\prime}(t)=-\beta(A t+B) e^{-\beta t}+A e^{-\beta t}
$$

y con los valores de las constantes, el resultado de Maple es

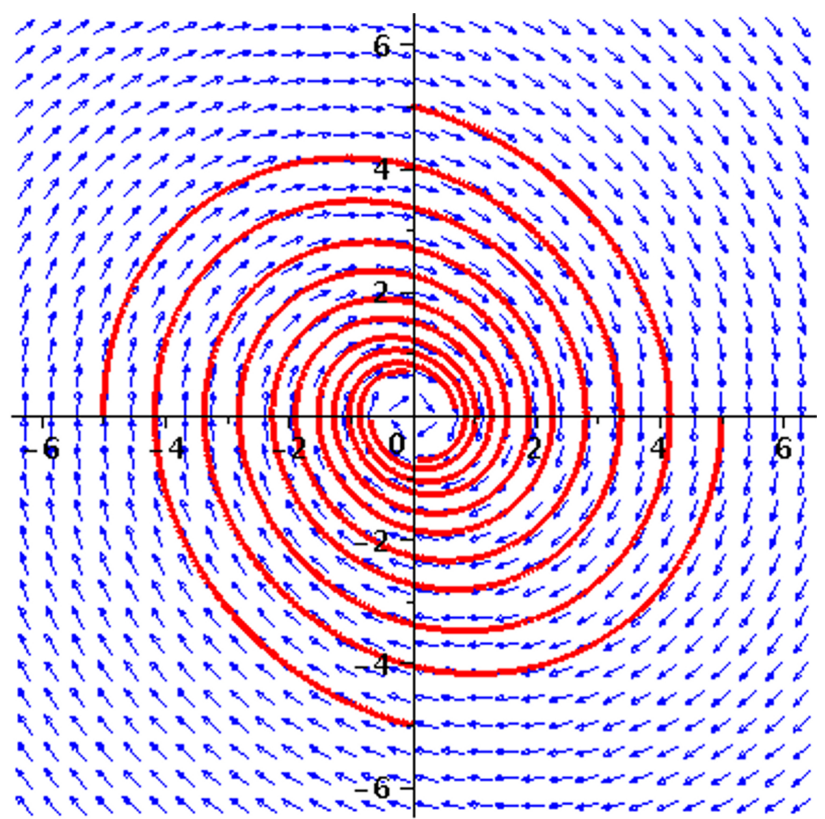

Figura 9: Diagrama de fase del movimiento subamortiguado.

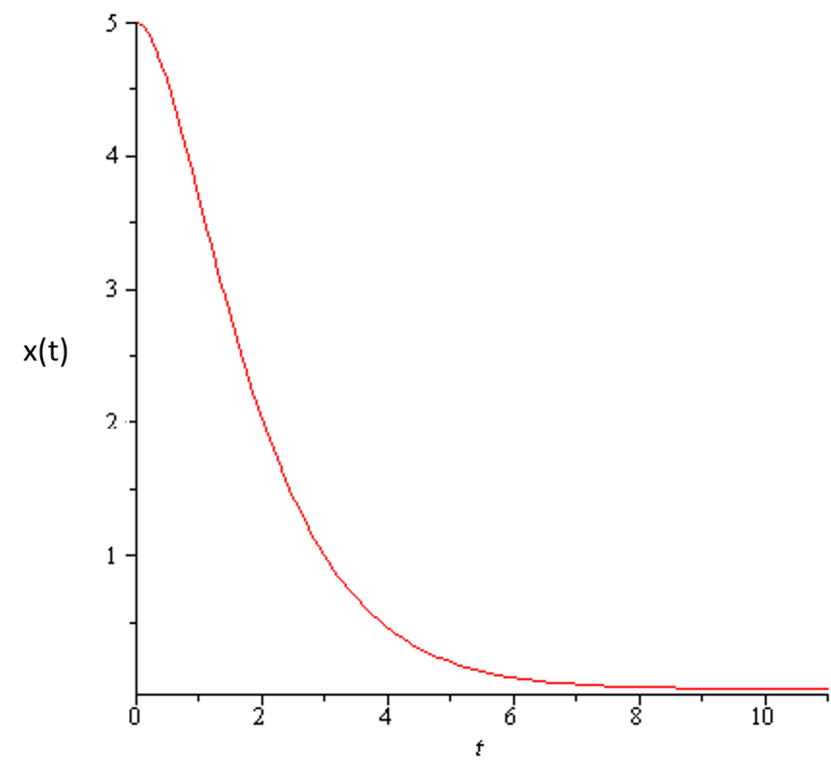

Figura 10: Posición en el movimiento críticamente amortiguado.

$$
\frac{d}{d t} x(t)=-5 e^{-t}(1+t)+5 e^{-t}
$$

En la Fig. 11, se aprecia que la velocidad tiende a un valor máximo negativo y luego a cero.

Para obtener el diagrama de fase, se puede realizar una transformación lineal en forma similar a la ec. (27),

$$
x^{\prime}(t)+\beta x(t)=A e^{-\beta t}
$$

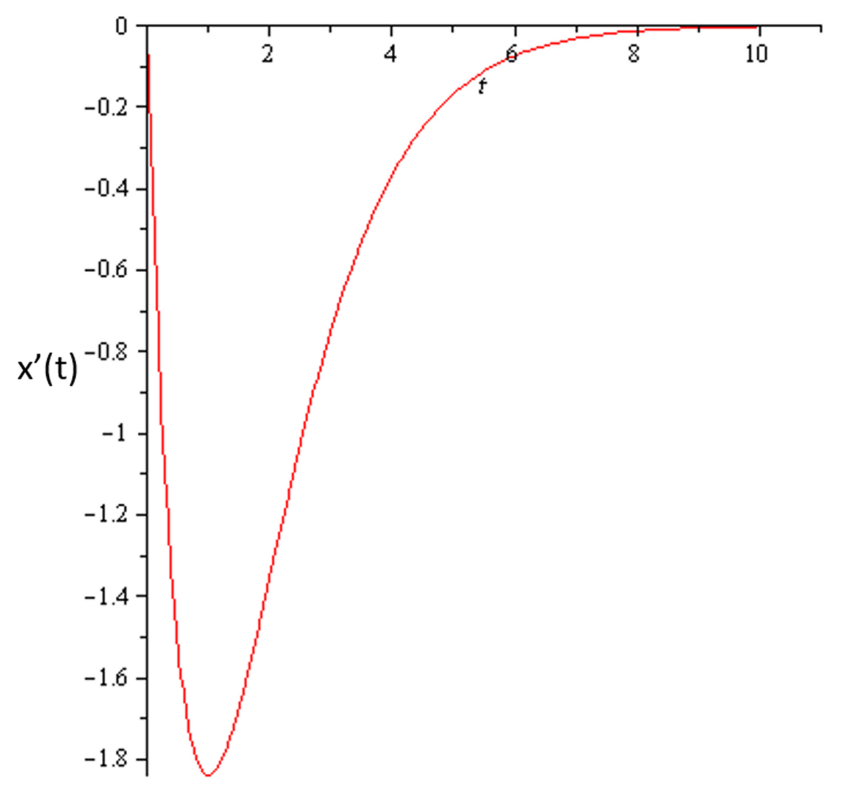

Figura 11: Velocidad en el movimiento críticamente amortiguado. 
de lo cual se desprende que la trayectoria de este movimiento en el espacio fase se aproxima a una recta con pendiente $-\beta$ que intersecta al origen. La Fig. 12 muestra el diagrama de fase generado por Maple para el movimiento críticamente amortiguado con diferentes condiciones iniciales.

\subsection{Oscilador sobreamortiguado}

Cuando $\beta>\omega_{0}$, se dice que el sistema es sobreamortiguado y su función de posición queda expresada como

$$
x(t)=C_{1} e^{\lambda_{1} t}+C_{2} e^{\lambda_{2} t},
$$

donde

$$
\lambda_{1,2}=-\beta \pm \omega_{d}
$$

y su función de velocidad es

$$
x^{\prime}(t)=-\beta x(t)+\omega_{d} e^{-\beta t}\left(C_{1} e^{\omega_{d} t}-A C_{2} e^{-\omega_{d} t}\right)
$$

Los resultados de Maple para la posición y la velocidad con valores $M=20 \mathrm{~kg}, k=10 \mathrm{~N} / \mathrm{m}, A=5 \mathrm{~m}$ y $c=30 \mathrm{~kg} / \mathrm{s}$, respectivamente, son

$$
x(t)=10 e^{-\frac{1}{2} t}-5 e^{-t}
$$

y

$$
\frac{d}{d t} x(t)=-5 e^{-\frac{1}{2} t}-5 e^{-t} .
$$

Las gráficas de 41] y 42 aparecen en la Fig. 13 y la Fig. 14, respectivamente.

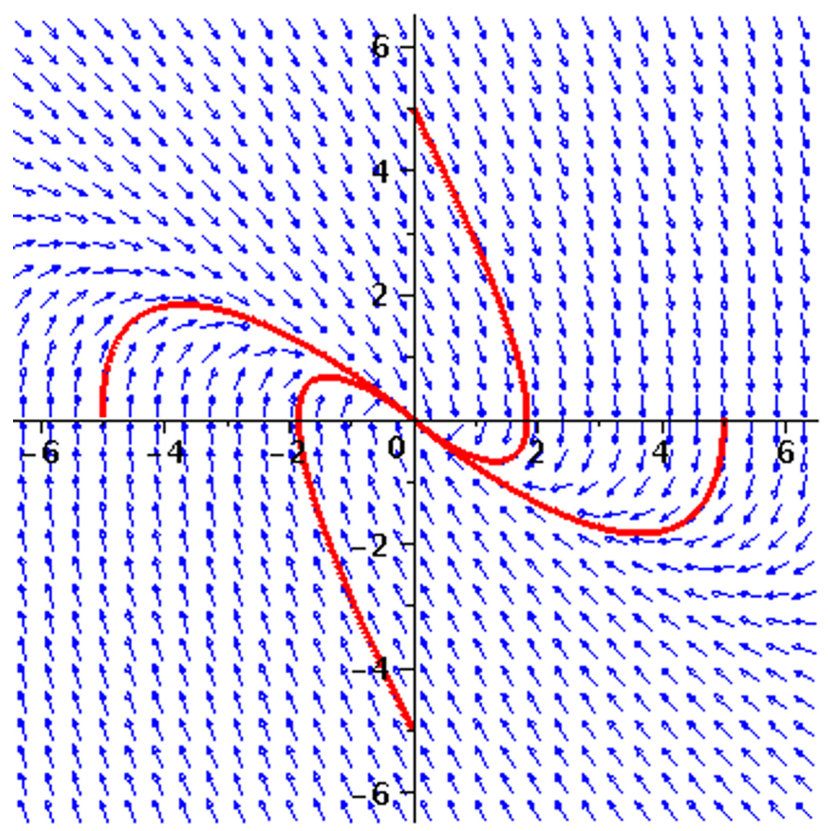

Figura 12: Diagrama de fase del movimiento críticamente amortiguado.

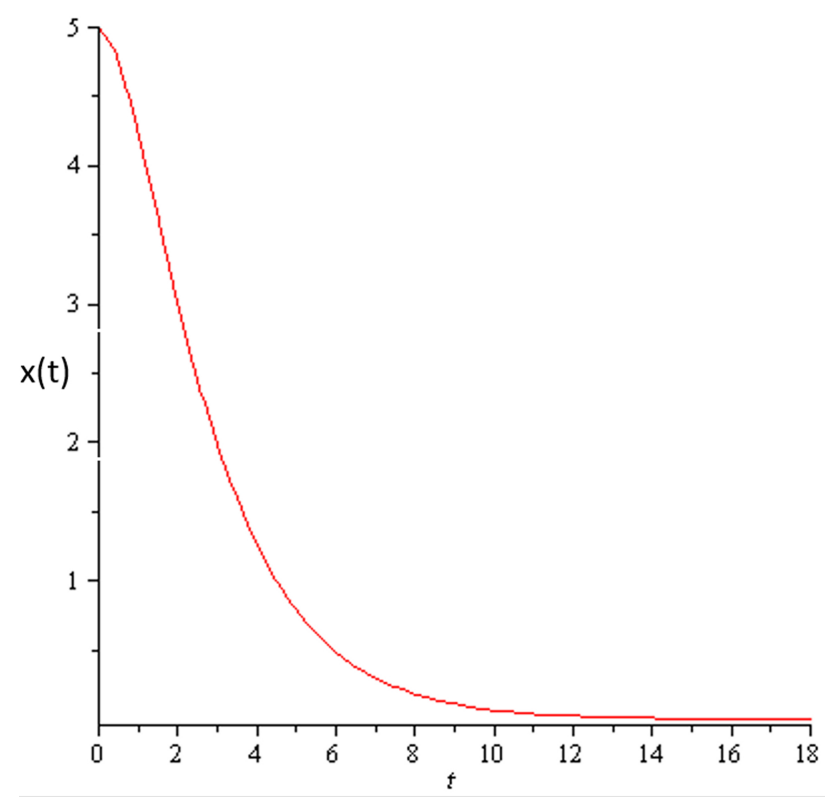

Figura 13: Posición del movimiento sobreamortiguado.

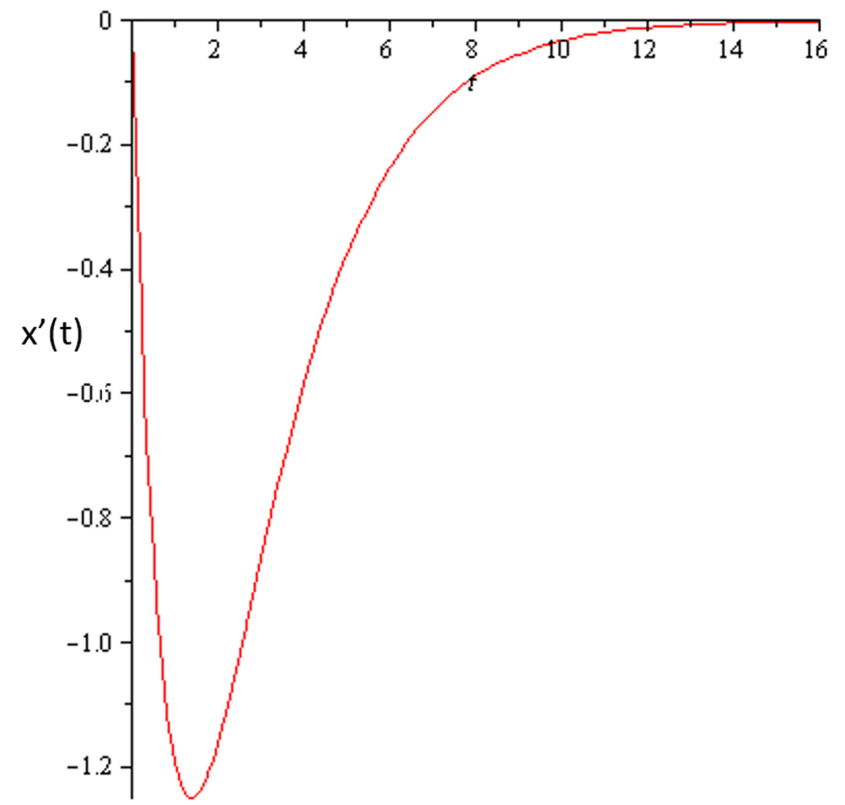

Figura 14: Velocidad del movimiento sobreamortiguado.

que se parecen a las del movimiento críticamente amortiguado, en el que la función de posición se aproxima más rápido a la posición de equilibrio, tal como lo hace la función de velocidad, por valores negativos. La Fig. 15 muestra cómo las trayectorias de fase intersectan al origen, pero más pronto que en el caso anterior. 


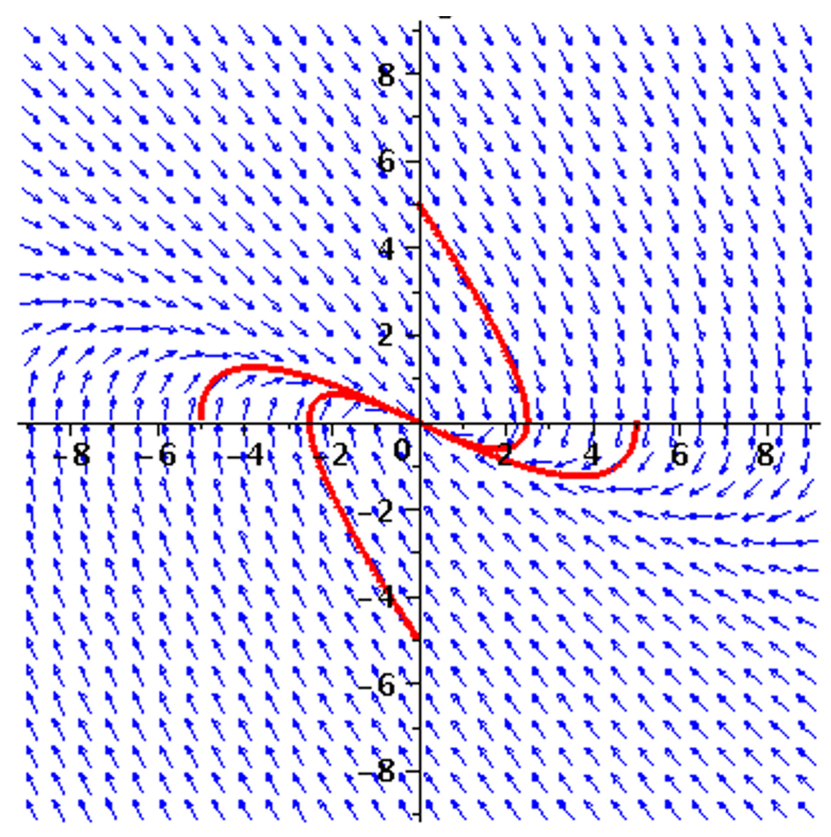

Figura 15: Diagrama de fase del movimiento sobreamortiguado.

\section{Comentarios}

López et al [15] señalan que, en la enseñanza de las ciencias, las actividades de simulación superan en número a las de modelación, pues estas requieren de conocimiento científico, de sus modelos matemáticos, así como de lenguaje de programación, con lo cual estamos de acuerdo. El sistema masa-resorte es un problema estudiado en los cursos básicos de física sin resolver las EDO involucradas [9, 10, 11], pues en ese momento aún no han tomado un curso de ED. El enfoque que hemos querido imprimirle a su estudio, en nuestros cursos de ED, incluye la modelación y la simulación asistidas por Maple para obtener respuestas de manera numérica, simbólica y gráfica. Sin estar familiarizados con el paquete computacional, los estudiantes generaron el modelo físico y matemático necesario para simular el comportamiento del sistema bajo diferentes condiciones a la que se les planteó como ejemplo. Como siguiente proyecto, propusimos el estudio del péndulo, sobre el cual ya trabajamos para someterlo a publicación.

Algunas ventajas de usar herramientas computacionales en este tipo de problemas que podemos mencionar son las siguientes:

1. Se apoya y motiva a los estudiantes a desarrollar soluciones formales a problemas prácticos, cercanos a los que deberán de resolver en asignaturas posteriores, como Circuitos Eléctricos o Vibraciones Mecánicas, sin recurrir a cálculos laboriosos.

2. Se estimula a los estudiantes no sólo a desarrollar una técnica y formulario en la solución de problemas, sino también la creatividad, el diseño y el sentido común.

3. Se permite hacer, a los estudiantes, un análisis de sensitividad al modificar los valores de los parámetros en que quedan expresadas las soluciones obtenidas.

Como parte del proceso de evaluación del trabajo final, establecimos dos rúbricas; una para evaluar el producto final y otra para evaluar la participación de los compañeros de cada equipo. Además de buenos resultados en el producto final, detectamos algunos fenómenos relacionados con habilidades, actitudes y valores como capacidad de trabajo, empatía, liderazgo, responsabilidad, entre otras, que son parte de la formación integral de los estudiantes.

\section{Apéndices}

\section{Apéndice A (Movimiento Armónico Simple)}

- Se reinicia el espacio de trabajo y se importan las librerías a utilizar.

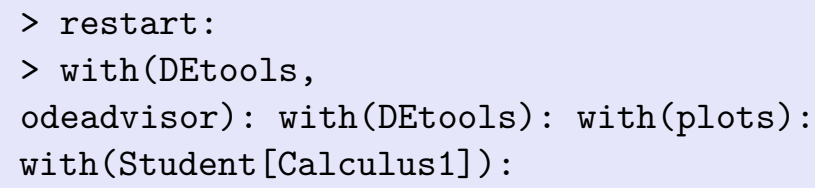

- Se establece la ecuación diferencial que modela al sistema masa-resorte.

$$
\begin{aligned}
& >\text { equ : } \\
& =m *(\operatorname{diff}(x(t), t \$ 2))+k * x(t)=0: \text { equ }
\end{aligned}
$$

- Se asignan valores a las constantes (m para masa, k para la constante del resorte y desp_In para el desplazamiento inicial)

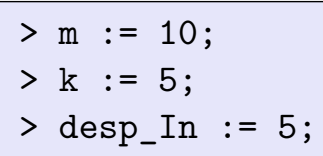

- Se sustituyen los valores de las condiciones iniciales. 


\section{$>$ equ;}

- Se simplifica el sistema dividiendo la ecuación entre el valor de la masa.

$>$ equ := equ/m: equ;

- Se resuelve la ecuación diferencial

$>$ sol_general := dsolve (equ): sol_ general;

- Se establecen las condiciones iniciales de la ecuación diferencial.
$>\mathrm{Xo}_{\mathrm{O}}:=\mathrm{desp}_{-} \mathrm{In}$;

$>$ Vo $:=0$;

- De acuerdo con las condiciones iniciales, se encuentra la solución particular.

$>$ sol_particular1 := dsolve( $\{$ equ, $\mathrm{x}(0)=\mathrm{Xo}, \mathrm{D}(\mathrm{x})(0)=\mathrm{Vo}\}, \mathrm{x}(\mathrm{t})): \mathrm{sol}_{-}$ particular1;

- Se determina el periodo del sistema.

$>$ periodo $:=(2 * \mathrm{Pi}) / \operatorname{sqrt}(\mathrm{k} / \mathrm{m})$;

- Se grafica la función de posición.

$>$ DEplot (equ, $x(t), t=-$ periodo $* 1.5$. periodo $* 1.5,[[x(0)=$

Xo, $\mathrm{D}(\mathrm{x})(0)=\mathrm{Vo}]$, color=[blue,blue], title="Solución del Movimiento Armónico Simple", stepsize $=0.01$, linecolour $=\sin (t * \mathrm{P} i)$, scaling $=$ constrained, titlefont $=$ [HELVETICA, BOLD, 12], symbol $=$ DIAMOND, resolution $=500$, axesfont $=$ [HELVETICA, BOLD, 10]);

- Se grafica el diagrama de fase del movimiento armónico simple.

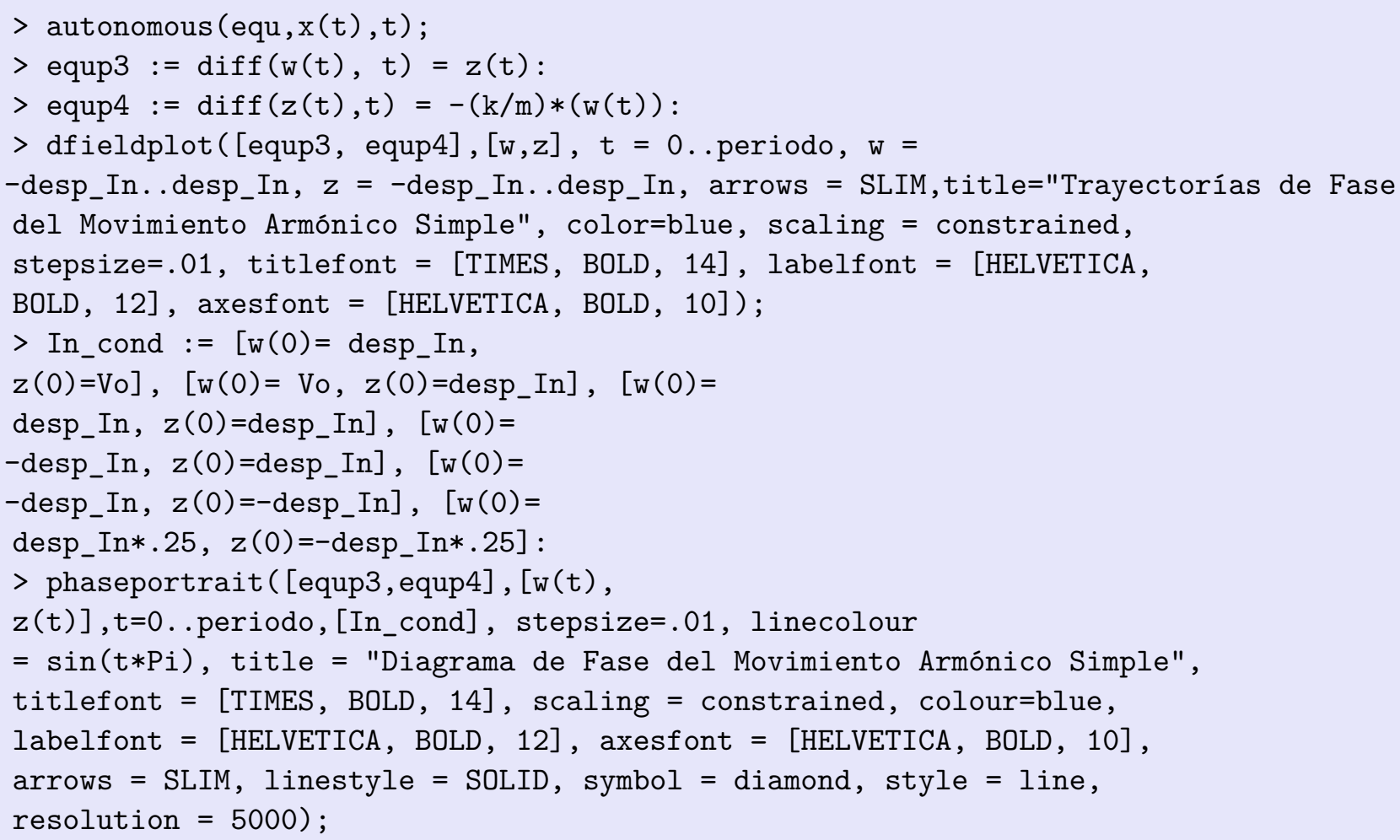




\section{Apéndice B (Movimiento con amortiguamiento)}

- Se reinicia el espacio de trabajo y se importan las librerías a utilizar.

restart:

with(DEtools): with(plots): with(plottools): with(Student [Calculus1]):

with (StringTools):

- Se establece la ecuación diferencial que modela el sistema del oscilador con amortiguamiento.

equ $:=m *(\operatorname{diff}(x(t), t \$ 2))+c *(\operatorname{diff}(x(t), t))+k * x(t)=0:$ equ;

- Se establecen los valores de las constantes.

$c:=$ (Inserte valor de condición inicial aquí);

$\mathrm{A}:=$ (Inserte valor de

condición inicial aquí);

$\mathrm{k}:=$ (Inserte valor de

condición inicial aquí);

$\mathrm{m}:=$ (Inserte valor de

condición inicial aquí);

Vo := (Inserte valor de

condición inicial aquí);

- Se sustituyen los valores en la ecuación diferencial. equ;

- Se divide la ecuación diferencial entre la masa.

equ $:=$ equ $/ \mathrm{m}$ : equ;

- De acuerdo con las condiciones iniciales y los valores de las constantes, se establece la frecuencia natural y el periodo del sistema.

omega := $\operatorname{sqrt}(\mathrm{k} / \mathrm{m})$;

tau $:=(2 * \mathrm{Pi}) /$ omega;

- Después de conocer el periodo y frecuencia natural del sistema, se identifica el tipo de amortiguamiento que tiene el oscilador, además de la solución general de su ecuación.

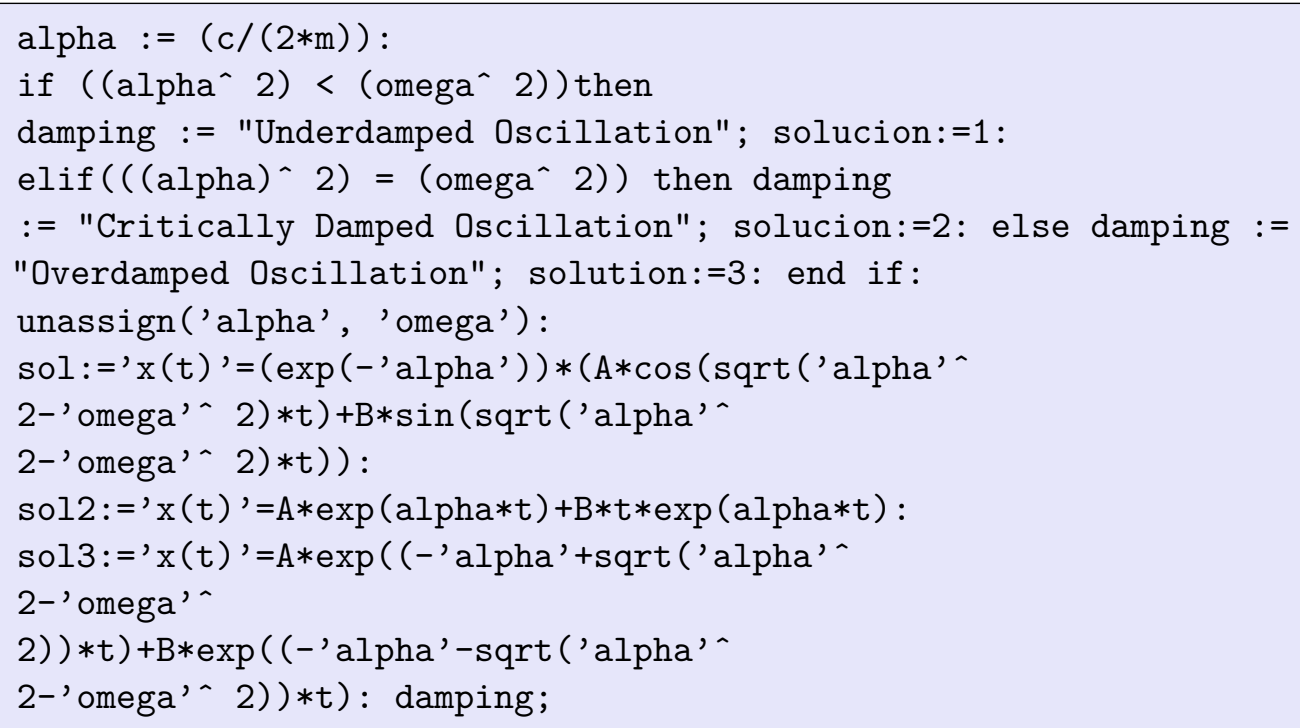


- Se obtiene una solución general de la ecuación diferencial.

res_gen := simplify(dsolVoe(equ)): res_gen;

- Con base en las condiciones iniciales, se establece la solución particular del sistema.

res_part := simplify(dsolVoe $(\{$ equ, $\mathrm{x}(0)=$

$A, D(x)(0)=V o\}, x(t)))$ : res_part;

dsolVoe $(\{@ \varrho(D, 2)(x)(t)+2 D(x)(t)+x(t)=0, x(0)=$

$5, D(x)(0)=0\}, x(t))$

- Se grafica la solución.

DEplot (equ, $x(t), t=0 . . t a u * 2.5,[[x(0)=A, D(x)(0)=V o]]$,

stepsize $=.005$, linecolour $=\sin (t * \mathrm{Pi})$, title $=$ Join (["Graph of the",

damping], " "), titlefont = [TIMES, BOLD, 14], colour=blue, labelfont

$=[$ HELVETICA, BOLD, 12], axesfont $=[$ HELVETICA, BOLD, 10], arrows =

SLIM, view=[-tau*.1..tau*3,-tau..tau $]$ );

- Se muestra el diagrama de fase para el movimiento con amortiguamiento.

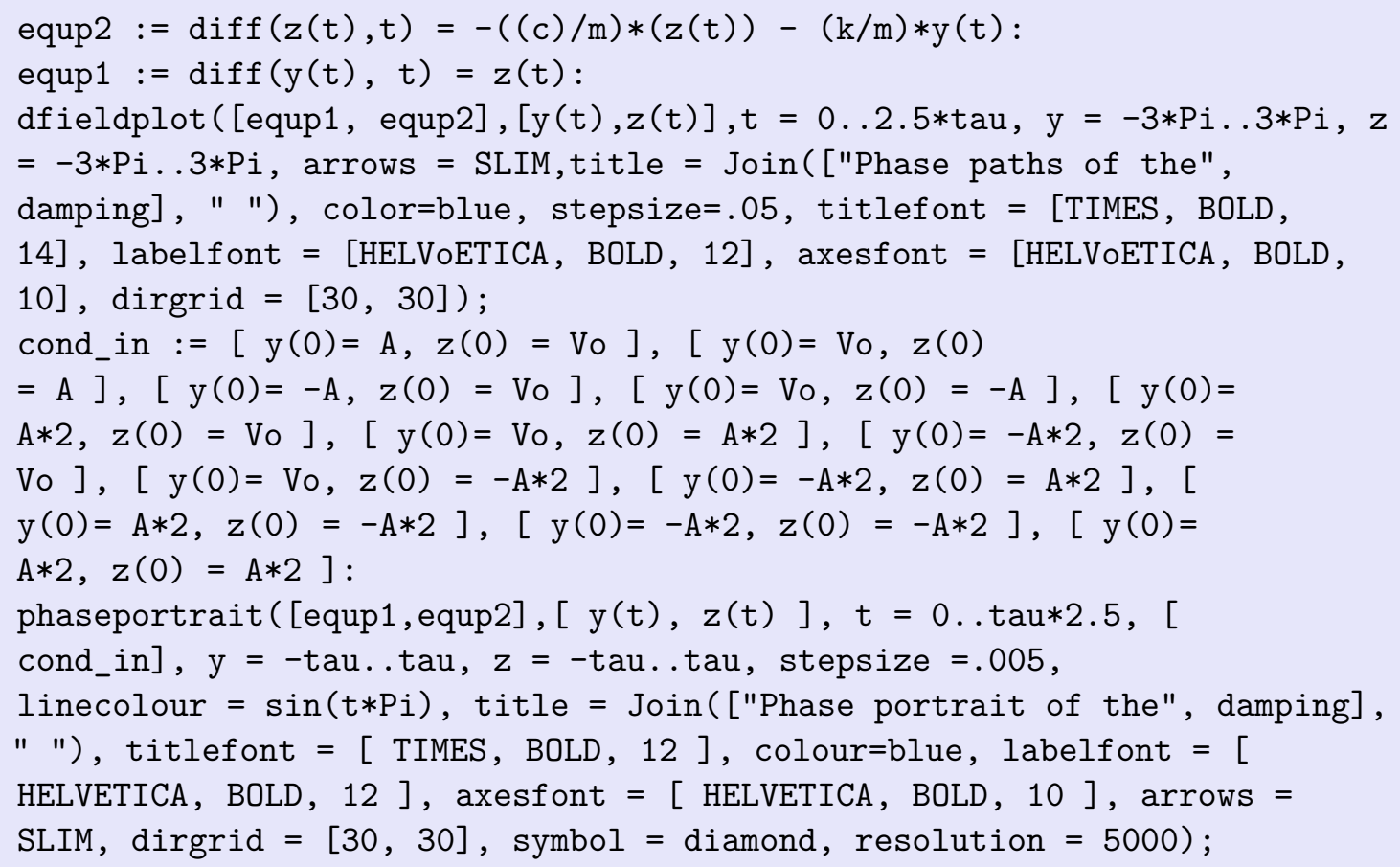




\section{Nota}

Para la obtención de las gráficas de velocidad, tanto del Movimiento Armónico Simple como del Amortiguado:

- Se copia y pega la solución de la ecuación diferencial en un nuevo archivo de MAPLE.

- Se da click derecho en la función y damos click en derivate $\rightarrow X$ para obtener la derivada de la función de posición, que es la velocidad.

- Se da click derecho en la función de la velocidad y click en Plots $\rightarrow 2$-D Plots.

\section{Referencias}

[1] E. Kreyszig, Advanced Engineering Mathematics (Wiley \& Sons, New York, 1972), 3rd ed.

[2] C.R. Wylie and L.C. Barret, Advanced Engineering Mathematics (McGraw-Hill, New York, 1995), 6th ed.

[3] P. Blanchard, R.L. Devaney and G.R. Hall, Differential Equations (Brooks Cole, Boston, 1998).

[4] D.G. Zill, Differential Equations with Computer Lab Experiments (Brooks Cole, Boston, 1998), 2nd ed.

[5] D.G. Zill and M.R. Cullen, Differential Equations with boundary-Value Problems (Brooks Cole, Boston, 2001), 5th ed.

[6] C.H. Edwards and D.E. Penney, Elementary Differential Equations with Applications (Pearson Education, New York, 2008), 6th ed.

[7] R.K. Nagle, E.B. Saff and A.D. Snider, Ecuaciones Diferenciales y Problemas con valores en la Frontera (Pearson, México, 2001), $3^{\mathrm{a} e d .}$

[8] G.F. Simmons, Differential Equations with Applications and Historical Notes (McGraw-Hill, New York, 1991), 2nd ed.

[9] R. Resnick, D. Halliday and K.S. Krane, Physics (John Wiley \& Sons, Hoboken, 2001), 7th ed.

[10] R.A. Serway, Physics for Scientists and Engineers with Modern Physics (Saunders College Publishing, Orlando, 1996), 4th ed.

[11] H.C. Ohanian and J.T. Market, Physics for Engineers and Scientists Vol. 1 (W.W. Norton \& Company, Inc., New York, 2007), 3rd ed.

[12] S.T. Thornton and J.B. Marion, Classical Dynamics of Particles and Systems, (Brooks Cole, Boston, 2004), 5th ed.

[13] R.I. Greene, Classical Mechanics with Maple (Springer, New York, 1995).

[14] F.E. Bueno Pascual y M.A. López-Mariño, in: XLVII Congreso Nacional de Física, Hermosillo, Sonora, (2004), page 157.

[15] S. López, E.A. Veit e I. Solano Araujo, Revista Brasileira de Ensino de Física 38, e2401 (2016). 